\title{
Improving diagnostic performances of peanut allergy using novel molecular components
}

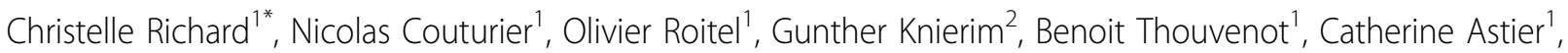 \\ Françoise Codreanu ${ }^{3}$, Gisèle Kanny ${ }^{4}$, Denise-Anne Moneret-Vautrin ${ }^{5}$, Bernard Bihain ${ }^{1}$, Sandrine Jacquenet ${ }^{1}$ \\ From 5th International Symposium on Molecular Allergology (ISMA 2013) \\ Vienna, Austria. 6-7 December 2013
}

Accurate in-vitro component-based diagnosis will allow clinicians to focus their efforts toward complex issues such as disease severity and reactive dose evaluation as well as establishing effective eviction measures. However to achieve this, clinical utility of component-resolved peanut allergy testing must be rigorously evaluated. In this pilot study, we compared performances of 2 component-resolved peanut allergy assays from Hycor ${ }^{\circledR}[\mathrm{H}]$ and Thermo Scientific ${ }^{\circledR}[\mathrm{T}]$ that are based on recombinant allergens (rAra h $1[\mathrm{H} / \mathrm{T}]$, rAra h $2[\mathrm{H} / \mathrm{T}]$, rAra h $3[\mathrm{H} / \mathrm{T}]$, rAra h $6[\mathrm{H}]$, rAra h $7[\mathrm{H}]$, rAra h $8[\mathrm{H} / \mathrm{T}]$ and rAra $h 9[\mathrm{H} / \mathrm{T}])$. IgE reactivity to each allergen was measured in 34 clinically characterized patients: 26 peanut-allergic and 8 atopic subjects from Eastern and Northern France. Total peanut extract assay $[\mathrm{T}]$ yielded low performances even with a $0.35 \mathrm{kU} / \mathrm{L}$ cut-off (sensitivity: $92 \%$, specificity: $25 \%$ ). Quantifications of IgE to rAra h 1, rAra h 2 and rAra h 3 show a strong correlation between the two manufacturers ( $\mathrm{rAra} \mathrm{h} 1: \mathrm{r} 2=$ 0.85, $\mathrm{p}<10-3$; rAra h 2: $\mathrm{r} 2=0.96, \mathrm{p}<10-3$; rAra h 3: $\mathrm{r} 2=0.90, \mathrm{p}<10-3)$ and achieve the same level of performances independently of the allergen source. Among these three assays, rAra $\mathrm{h} 2[\mathrm{H} / \mathrm{T}]$ yielded a sensitivity of $85 \%$ and a specificity of $100 \%$ with a $0.35 \mathrm{kU} / \mathrm{L}$ cut-off. The rAra h 6 assay is available from Hycor ${ }^{\circledR}$ only and yielded a sensitivity of $92 \%$ and a specificity of $100 \%$ using the same cut-off, i.e. $24 / 26$ peanut allergic patients are positive for $\mathrm{rAra} h 6$. The IgE from the 2 undiagnosed patients do not react with any of the other recombinant from any sources. Neither Hycor ${ }^{\circledR}$ nor Thermo Scienti$\mathrm{fic}^{\circledR}$ rAra h 8 assays are clinically useful for peanut allergy diagnosis in spite of their specificity differences at $50 \%$ and $25 \%$ respectively. Thermo Scientific $^{\circledR}$ rAra h 9 assay was not clinically useful with no positive sample detected. Hycor $^{\circledR}$ rAra h 9 assay detected 2 of the 26 peanut allergic patients without reactivity in the atopic patients (specificity: $12 \%$, sensitivity: $100 \%$ ). This is consistent with the low prevalence of Ara $\mathrm{h} 9$ sensitisation in Northern Europe. This study suggests the possibility of improving the clinical utility of molecular component assays by substituting rAra h 6 to rAra h 2. It also introduces rAra h 9 as a novel component that might be a significant clinical value in countries outside Northern Europe. This will be evaluated in multicenter studies.

\section{Authors' details}

${ }^{1}$ GENCLIS SAS, Allergy Department, Vandoeuvre-Les-Nancy, France. ${ }^{2}$ Hycor, Allergy Department, Kassel, Germany. ${ }^{3}$ Centre Hospitalier de Luxembourg, Food Allergy Department, Luxembourg, Luxembourg. ${ }^{4}$ Nancy Hospital, Food Allergy Department, Nancy, France. ${ }^{5}$ Epinal Hospital, Food Allergy

Department, Epinal, France.

Published: 17 March 2014

doi:10.1186/2045-7022-4-S2-P43

Cite this article as: Richard et al.: Improving diagnostic performances of peanut allergy using novel molecular components. Clinical and Translational Allergy 2014 4(Suppl 2):P43. 